\title{
Tidal disruption of planetary bodies by white dwarfs - II. Debris disc structure and ejected interstellar asteroids
}

\author{
Uri Malamud $^{\oplus 1,2 \star}$ and Hagai B. Perets ${ }^{\oplus 1,3}$ \\ ${ }^{1}$ Department of Physics, Technion - Israel Institute of Technology, Technion City, 3200003 Haifa, Israel \\ ${ }^{2}$ School of the Environment and Earth Sciences, Tel Aviv University, Ramat Aviv, 6997801 Tel Aviv, Israel \\ ${ }^{3}$ TAPIR, California Institute of Technology, Pasadena, CA 91125, USA
}

Accepted 2020 January 12. Received 2020 January 9; in original form 2019 November 21

\begin{abstract}
We make use of a new hybrid method to simulate the long-term, multiple-orbit disc formation through tidal disruptions of rocky bodies by white dwarfs, at high-resolution and realistic semimajor axis. We perform the largest yet suite of simulations for dwarf and terrestrial planets, spanning four orders of magnitude in mass, various pericentre distances, and semimajor axes between 3 and 150 au. This large phase space of tidal disruption conditions has not been accessible through the use of previous codes. We analyse the statistical and structural properties of the emerging debris discs, as well as the ejected unbound debris contributing to the population of interstellar asteroids. Unlike previous tidal disruption studies of small asteroids which form ring-like structures on the original orbit, we find that the tidal disruption of larger bodies usually forms dispersed structures of interlaced elliptic eccentric annuli on tighter orbits. We characterize the (typically power law) size distribution of the ejected interstellar bodies as well as their composition, rotation velocities, and ejection velocities. We find them to be sensitive to the depth (impact parameter) of the tidal disruption. Finally, we briefly discuss possible implications of our results in explaining the peculiar variability of Tabby's star, the origin of the transit events of ZTF J0139+5245 and the formation of a planetary core around SDSS J1228+1040.
\end{abstract}

Key words: hydrodynamics - white dwarfs - transients: tidal disruption events.

\section{INTRODUCTION}

Between 25 percent and 50 per cent of all WDs (Zuckerman et al. 2003, 2010; Koester, Gänsicke \& Farihi 2014) are found to be polluted with heavy elements. The existence of orbiting gas and dust as deduced from measurements of infrared excess and metal emission lines are thought to originate from accretion of planetary material (Debes \& Sigurdsson 2002; Jura 2003; Kilic et al. 2006; Jura 2008). In particular, the inferred composition of both WD atmospheres (Wolff, Koester \& Liebert 2002; Dufour et al. 2007; Desharnais et al. 2008; Klein et al. 2010; Gänsicke et al. 2012; Jura \& Young 2014; Harrison, Bonsor \& Madhusudhan 2018; Hollands, Gänsicke \& Koester 2018; Doyle et al. 2019; Swan et al. 2019) and their discs (Reach et al. 2005; Jura et al. 2007; Jura, Farihi \& Zuckerman 2009; Reach et al. 2009; Bergfors et al. 2014; Farihi 2016; Manser et al. 2016; Dennihy et al. 2018) suggests that the polluting material is terrestrial-like and typically dry.

Such material was suggested to originate from planetary bodies which are perturbed by some mechanism (Debes \& Sigurdsson

^E-mail: urimala@physics.technion.ac.il
2002; Bonsor, Mustill \& Wyatt 2011; Debes, Walsh \& Stark 2012; Kratter \& Perets 2012; Perets \& Kratter 2012; Shappee \& Thompson 2013; Michaely \& Perets 2014; Stone, Metzger \& Loeb 2015; Veras \& Gänsicke 2015; Hamers \& Portegies Zwart 2016; Payne et al. 2016; Veras 2016; Caiazzo \& Heyl 2017; Payne et al. 2017; Petrovich \& Muñoz 2017; Stephan, Naoz \& Zuckerman 2017; Smallwood et al. 2018) to highly eccentric orbits with proximity to the WD, and are subsequently tidally disrupted to form a circumstellar disc of planetary debris.

Neither the original processes that form debris discs around WDs (Veras et al. 2014), nor the subsequent processes that generate a more compact accretion disc (Veras et al. 2015) nor the actual accretion processes on to the WD (Jura 2008; Rafikov 2011; Metzger, Rafikov \& Bochkarev 2012) are entirely understood. The former in particular is still very much an open question. We do not know how the original debris disc initially forms, or in what way are the debris properties affected by the orbit, eccentricity, size, and composition of the tidally disrupted progenitor. We are also driven by recent discoveries of disintegrating minor planets with short (Vanderburg et al. 2015) as well as much longer (Vanderbosch et al. 2019) periodicity, the possible leftover core of a planet whose outer layers have been removed (Manser et al. 2019). These discoveries 
have generated a wide interest that extends beyond the white dwarf and planet-formation communities, and which emphasize the potential importance of understanding the initial stages of tidal disruption and debris disc formation.

To date, there exist very few detailed simulations of disc formation through tidal disruptions by WDs (Debes et al. 2012; Veras et al. 2014), and these are limited in resolution and length due to the computational expense of the hydrodynamical/N-body simulations involved. In an accompanying paper (Malamud \& Perets 2020; hereafter Paper I), we have presented a novel hybrid hydrodynamicalanalytical approach that can resolve such difficulties. In this paper, we utilize the code to perform the largest-yet suite of simulations of tidal disruptions of dwarf and terrestrial planets by WDs, covering a large set of orbital set-ups, and a very wide range of masses up to terrestrial planet size. This phase space probes for the first time the formation sequence, consequences, and ensuing properties of debris discs that emerge in such tidal disruptions. Without the hybrid model, such initial conditions were never before accessible through the use of any previous code.

In Paper I, we discuss the distinctive difference between debris discs of small versus large disrupted planetesimals. Given characteristic Solar system distances, small asteroids usually form a narrow ring in which all the asteroid material is bound to the star and remains on semimajor axes close to that of the original progenitor (in similarity to the Veras et al. 2014 study). Such disruptions are therefore termed 'non-dispersive regime'. In contrast, larger dwarf or terrestrial-sized planets usually form a completely different debris disc, in which just over half of the material becomes tightly bound to the star (compared to the original progenitor orbit) and the other half becomes unbound. Such disruptions are therefore termed 'bi-modal regime'. This paper is the first to present detailed simulations of the latter type.

The layout of the paper is structured as follows: in Section 2, we briefly review our hybrid approach, and the criteria for selecting a suite of hybrid simulations of tidal disruptions by WDs, spanning a large range of masses, semimajor axes and pericentre distances. In Section 3, we track the formation process of the ensuing debris discs, characterizing their typical formation time-scales, and discussing their statistical properties, including fragment orbits, sizes, and rotations. In Section 4, we track the ejected unbound debris that forms interstellar asteroids. We likewise discuss the statistics of their size distribution, rotation, and velocity. In Section 5, we briefly discuss possible implications and future work regarding the origin of the variability of Tabby's star, the transiting object ZTF J0139+5245, and the formation of what could be a planetary core remnant in SDSS J1228+1040, potentially arising from tidal disruptions of dwarf or terrestrial-sized planets. Finally, in Section 6, we summarize the paper's main points.

\section{THE HYBRID APPROACH: A STUDY OF TIDALLY DISRUPTED DWARF AND TERRESTRIAL PLANETS}

\subsection{Outline}

The hybrid approach is intended for an efficient treatment of disc formation by tidal disruptions. The details of this approach are introduced and discussed in detail in Paper I. Here, we only provide a brief review of the main ideas.

The approach makes use of the fact that the primary processes taking place during these tidal disruptions are restricted to a relatively small spatial domain. First, we recall that the differential gravitational force that breaks the object apart is relevant only to the Roche limit of the star. The second important phase is fragmentation. It is during this phase that small particles in the tidal stream may collapse by the stream's own self-gravity, to form larger fragments. The relevant spatial domain here, as discussed in Paper I, is also rather small, exceeding the tidal sphere only by an order of magnitude or so. Overall, the breakup and fragmentation phases constitute only a tiny fraction of the total spatial domain (of the original orbit), and are confined to the immediate environment of the star.

Our approach is therefore to restrict the SPH computations only to this relatively small domain, and to omit unnecessary calculations outside of it. Following the fragmentation phase, we identify the emerging fragments (whose constituent particles form spatially connected clumps of material), and for the reminder of their orbits, their trajectories are calculated and tracked analytically, assuming Keplerian orbits. Our hybrid program simply places each fragment once again near the star's Roche limit, based on its return orbital elements. This approach computationally outperforms full SPH modelling by at least a few orders of magnitude, and the benefit increases for more eccentric orbits. We make the assumption that the disc of debris is largely collisionless (shown to be accurate at the 99 per cent level in Paper I), as well as dynamically unaffected by radiation or other processes. The returning fragment immediately undergoes an additional tidal disruption, potentially splitting into a new set of fragments, with their own unique dispersion in orbital parameters, and so on, in an iterative process.

The hybrid code's main task is to identify the fragments, accurately calculate their orbits and especially handle the synchronization and timing of the subsequent disruptions. Apart from this, its other procedural task is handling the SPH job dissemination. The hybrid code terminates when reaching one of two outcomes: either all fragments have ceased disrupting given their exact size, composition, and orbit; or fragment disruption is inhibited when reaching its minimum size - that of a single SPH particle.

\subsection{Simulation suite and code setup}

The only previous work to simulate tidal disruptions and disc formation around WDs, considered small, kilometre-sized asteroids (Veras et al. 2014). Using the same modified $N$-body simulation model and a similar setup, disruptions of larger objects around a WD were also investigated by the same authors (Veras et al. 2017), however they do not discuss the formation of a disc or its emerging features, but rather focus on the rate of mass shedding of a differentiated planetesimal. Here, we perform a complimentary investigation, studying for the first time objects that range in size from small dwarf planets to terrestrial-sized planets, while focusing on the emerging debris discs. We note that the tail in the mass distribution function observed in WD atmospheres (see fig. 6 in Veras 2016) does not currently reach beyond the mass of a typical dwarf planet, however this could merely be an observational constraint. Certainly the large and dense orbiting object from Manser et al. (2019) implies that more massive planet or dwarfplanet disruptions are a possibility.

For our suite of simulations, whose results are summarized in Sections 3 and 4 below, we consider multiple pericentre distances: $0.1,0.5$, and $1 R_{\odot}$. The significance of the pericentre distance is discussed in length in Paper I, where it was shown that a large pericentre distance close to the Roche limit, results in a partial disruption, shedding only a small fraction of the planet's mass, emanating from its outer portions. Whereas if the pericentre is 
Table 1. Hybrid suite of simulations.

\begin{tabular}{lccc}
\hline Solar system analogue & Mass $\left(\mathrm{M}_{\oplus}\right)$ & $a(\mathrm{au})$ & $q\left(\mathrm{R}_{\odot}\right)$ \\
\hline Earth & 1 & 3 & $0.1,0.5,1$ \\
Mars & 0.1 & 3 & $0.1,0.5,1$ \\
Main-belt (Vesta) & 0.0001 & 9 & 1 \\
Satellite (Io) & 0.01 & 17 & 1 \\
Kuiper-belt (Eris) & 0.001 & 150 & 1 \\
\hline
\end{tabular}

Note. The listed masses are roughly compatible with our Solar system analogues. The semimajor axis $a$ is estimated assuming orbital expansion of approximately thrice the original orbit, and the pericentre distance $q$ is given in units of the WD's Roche radius.

halved, the disruption is full. It forms a classic narrow stream of debris, however the stream is gravitationally self-confined, and it collapses to form multiple fragments, which later also collide and merge among themselves, forming smaller second-generation particles. If the pericentre is merely a small fraction of the Roche limit, the disruption is both full and violent, such that the stream is gravitationally unconfined and the planet becomes almost entirely disassociated into its constituent particles.

Here, we focus primarily on partial disruptions from large pericentre distances for the following reasons. As mentioned in Section 1, numerous possibilities have been suggested regarding the mechanisms that inject planetesimals to tidal crossing orbits. At our current level of understanding, however, it is not yet possible to quantify their relative importance. Hence, we cannot estimate what choice of $q$ is physically more judicious or probable than any other, arbitrary choice. Generally speaking, studies of planetesimal injection rates (through the use of loss-cone formalism, e.g. Rickman et al. 2008, Grishin \& Veras 2019, and references therein) in the fast diffusion, full loss-cone regime suggest a linear dependence on the closest approach (Perets \& Gualandris 2010). However, in the empty loss-cone regime, planetesimals diffuse slowly and therefore typically go through Roche grazing orbits before further diffusing into more radial orbits. In other words, at least in the empty loss-cone regime, deeper disruptions are more depleted compared with the full loss-cone regime, and our choice to focus on large $q$ therefore gains more credibility. Finally, based on Paper I, we know that the hybrid model performs very well for large $q$. If one is to choose arbitrarily, it is plausible to focus on a high value of $q$, since the disc formation cannot otherwise be studied by any other means or existing models.

The fiducial WD mass in all of our simulations is $0.6 \mathrm{M}_{\odot}$, a common choice in many WD studies (Veras et al. 2014). We consider the objects listed in Table 1, as approximate Solar system analogues of dry composition, noting that we are not at all concerned with simulating their precise physical properties. We merely wish to cover a large range of masses and orbits, varying by several orders of magnitude.

For our Solar system analogues, we select Earth (terrestrialsized), Mars (planetary embryo sized), Vesta (main-belt rocky asteroid), Io (rocky satellite), and Eris (rocky Kuiper-belt object). Coincidentally, their masses vary by approximately one order of magnitude each, covering four orders of magnitude relative to the Earth's mass.

The distances listed in Table 1 are the final semimajor axes during the WD stellar evolutionary phase. At $a=3$ au, the orbit roughly corresponds to the maximum stellar radius of an $\sim 2 \mathrm{M}_{\odot}$ star (a $0.6 \mathrm{M}_{\odot}$ WD progenitor) during the AGB phase (Mustill \& Villaver 2012). A planetesimal orbiting at 1 au will undergo orbital expansion of a factor of $\sim 3(2 / 0.6)$, hence it has the potential to survive to the WD stage. We thus consider 3 au to be the lowest possible semimajor axis permitted for these simulations. According to more detailed analyses by Mustill \& Villaver (2012), Earth-like planets undergoing adiabatic orbital expansion may only survive engulfment by a $2 \mathrm{M}_{\odot}$ thermally pulsating AGB star when their initial orbit is beyond about 2 au. Hence, they are more likely to have a final semimajor axis of 6-7 au. We further note that orbital decay by tidal interaction during the much longer RGB phase is not important for terrestrial planets, since according to previous studies they do not experience strong tidal forces as do gas giants (Villaver et al. 2014) (but the gas-giant migration can still affect the orbital stability of smaller terrestrial planets). Here, we shall ignore all such complexities and simply select the smallest, physically permitted semimajor axis of 3 au, to be used as the minimum semimajor axis throughout this paper. The subsequent semimajor axes in Table 1 therefore correspond to the main-sequence orbits of $1,2.7,5$, and $45 \mathrm{au}$, respectively.

The details concerning the MILUPHCUDA SPH code setup, which is used to model the disruptions in the hybrid model, are identical to those used in the full SPH simulations in Paper I (see details therein). For simplicity, we assume the same 'terrestrial-like' composition and structure for all the analogue objects listed in Table 1. I.e., they are all composed of a rocky mantle and an iron core (with 70 per cent and 30 per cent mass fractions, respectively), despite obvious differences between them that might render their composition, in reality, rather dissimilar (especially the Kuiper-belt analogue).

Finally, the resolution used in the hybrid model for this study ranges between $50 \mathrm{~K}$ and $500 \mathrm{~K} \mathrm{SPH}$ particles. It is orders of magnitude higher than that used in the full SPH simulations discussed in Paper I, as indeed any other previous disc formation study. In most of the simulations below we use a nominal resolution of $200 \mathrm{~K}$ SPH particles.

\section{DISC FORMATION AND PROPERTIES}

\subsection{Semimajor axis distribution}

We analyse the histogram distribution of the semimajor axis $a$ for various simulations. In Fig. 1, we first look at simulations of Earth-sized planets, comparing their histograms (normalized by the total number of disrupted fragments) for different values of the pericentre distance $q$. Here, the planet's original semimajor axis is $3 \mathrm{au}$, and the WD mass is $0.6 \mathrm{M}_{\odot}$. We note that these histograms capture the debris state at the end of the simulations. Hence, they may represent one major disruption event, as in the case of panel 1(a); a superposition of the planet disruption and a multitude of fragment disruptions, corresponding to the full yet gravitationally self-confined state represented in panel 1(b); or a superposition of several partial disruptions that rip only of the outer portions of the original planet, and eventually the entire planet, as in panel 1(c). Such distinctions are important for our interpretation of the histogram results, and in Section 3.2, we further discuss the temporal evolution.

In Paper I, we introduce an analytical impulse approximation model, where we denote the planetesimal radius as $R$, and the displacement of its constituent particles as $r$, where $r_{\text {crit }}$ is the critical displacement from the planetesimal's centre of mass in the opposite direction of the WD, beyond which all constituent particles will become unbound after the disruption. For an Earthlike planet, $r_{\text {crit }} \ll R$ is a reasonable approximation. Hence, we can associate such disruptions with the bi-modal disruption regime (see 


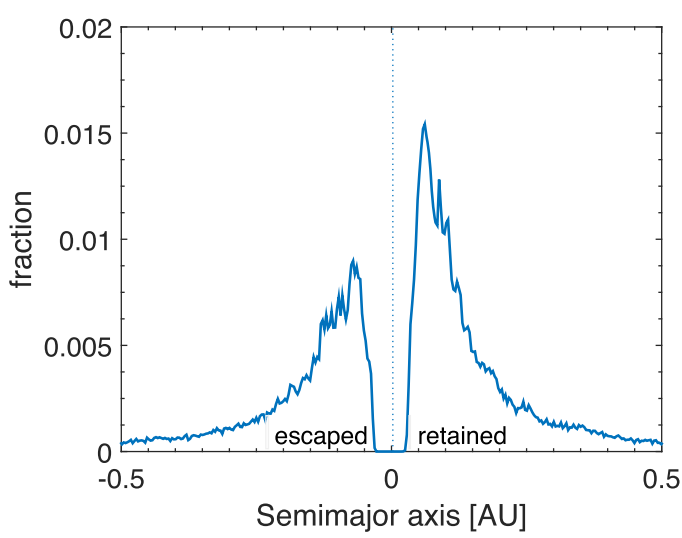

(a) $\mathrm{q}=0.1 R_{\odot}$

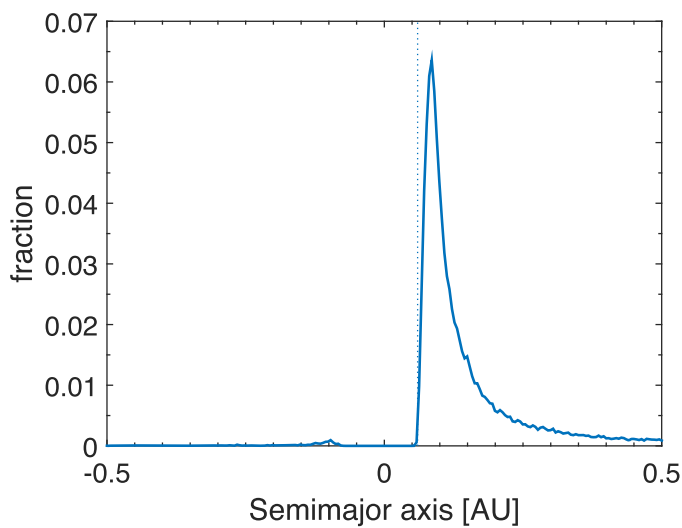

(b) $\mathrm{q}=0.5 R_{\odot}$

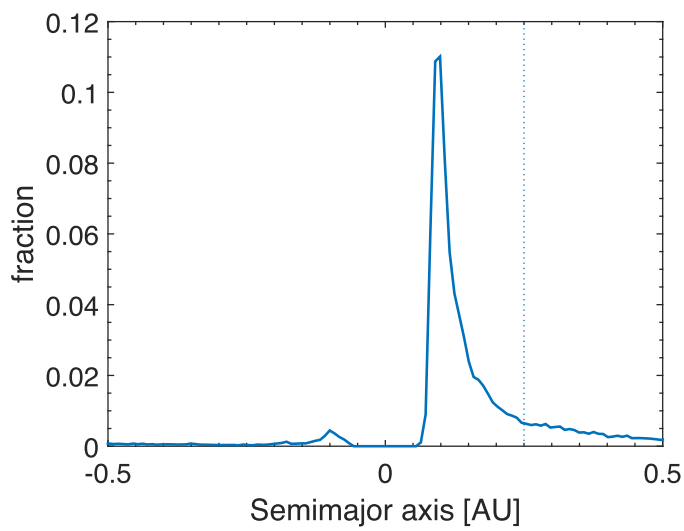

(c) $\mathrm{q}=1 R_{\odot}$

Figure 1. Semimajor axis normalized histogram of the final debris disc, formed by the tidal disruption of an Earth-sized planet around a $0.6 \mathrm{M}_{\odot}$ WD. The planet original semimajor axis is $3 \mathrm{au}$, whereas its pericentre distance $q$ is (a) 0.1 , (b) 0.5 , and (c) $1 \mathrm{R}_{\odot}$, respectively. Resolution is $200 \mathrm{~K}$ particles. Fragments retained $(a>0)$ or escaped $(a<0)$ in the disruption are labelled in panel (a). The theoretical impulse approximation model limits for the innermost semimajor axis are shown by vertical dotted lines. For a top-down view of the corresponding (bound) disc layout and temporal evolution see Paper I, Fig. 8.

Section 1), and calculate the tightest (innermost) semimajor axis of the disrupted constituent particles (á) in the ensuing disc according to the analytical model. In the simple impulse approximation view, the innermost $a$ arises from the radial separation of the particles on the planet's surface, and equals $d^{2} / 2 R$, where $d$ is the breakup distance. Plugging in the relevant numbers, and replacing $d$ for $q$, only the inner cut-off in panel 1(b) agrees with the analytical expectations (dotted vertical lines), as the innermost semimajor axis is calculated to be $\sim 0.06$ au, just like in the plot. For the deep disruption the analytical calculation leads to $\sim 0.0025 \mathrm{au}$, whereas panel 1(a) cuts-off at a semimajor axis around 10 times larger. For the grazing disruption, the analytical model leads to $\sim 0.25 \mathrm{au}$, whereas panel 1(c) cuts-off at a semimajor axis of only $0.05 \mathrm{au}$, around 5 times smaller.

The reason for this discrepancy is not trivial. We suggest three potential causes. The impulse approximation analytical model assumes that at the moment of breakup, the body's constituent particles instantaneously disassemble from the spherical rigid body, and instead form a swarm of particles with the same velocity as prior to the breakup $(v=v)$, but now evolve independently according to their spatial distribution. In reality, even if we assume the planet to be non-rotating prior to the disruption, simulations suggest that none of these assumptions is exactly correct. (a) The precise moment of breakup is difficult to determine, but it may actually precede the moment of closest approach (hence affecting $d$ ); (b) The breakup is not instantaneous, and thus to some extent the object deviates from sphericity when this moment is reached (hence affecting $R$ ); (c) The non-instantaneous transformation from the gravity-dominated to the disassembled state also gives the particles an extra spin, thus deviating from the underlying assumption that $v=v$ in the analytical model.

If the planet is also rotating prior to entering the tidal sphere, these points are amplified. How can this explain the results in Fig. 1? The cut-off in panel 1(a) suggests that a combination of a large planet and a deep disruption expedite the moment of breakup. Simply put, the planet disrupts prior to the moment of closest approach, which decreases the semimajor axis dispersion. In panel 1(c), the situation is rather different. The disruption is partial, and only the outermost particles disassemble from the rest of the planet. Additionally, here the planet deforms slowly and the radial distance of these particles during breakup likely increases, in addition to obtaining an extra spin. Both of these effects increase the semimajor axis dispersion and lead to a smaller cut-off than predicted by the analytical model. Finally, panel 1(c) depicts the state at the end of the evolution, whereas the first flyby results in a much more modest dispersion (cut-off at $\sim 0.15 \mathrm{au}$ ), and only after the second flyby of the planet do we reach the final $\sim 0.05$ au cut-off. The previously mentioned effects are most likely amplified by the newly gained fast-rotation of the planet prior to entering the tidal sphere for the second time.

Interestingly, for Fig. 1, the overall outcome in the semimajor axis dispersion turns out to be similar, despite three, very different disruption modes. This relatively constant outcome in varying $q$ corroborates previous results for stellar disruptions that also found an approximate constancy in the (equivalent) energy spread (Guillochon \& Ramirez-Ruiz 2013; Stone, Sari \& Loeb 2013; Steinberg et al. 2019). To our knowledge, this paper is the first to suggest the same in planetary disruptions.

Lastly, we note that none of the inner cut-offs is as small as that of the disintegrating minor planet around WD 1145+017. At $\sim 0.005$ au (Vanderburg et al. 2015), its orbit is one-fifth as small as the smallest value in panel 1(a), which could be another strong piece of evidence that this object has undergone further evolution that tightened and circularized its orbit.

Fig. 1 also features a clear asymmetry between the bound and unbound (negative semimajor axis) material, which could be easily explained by repeated disruptions. According to the 


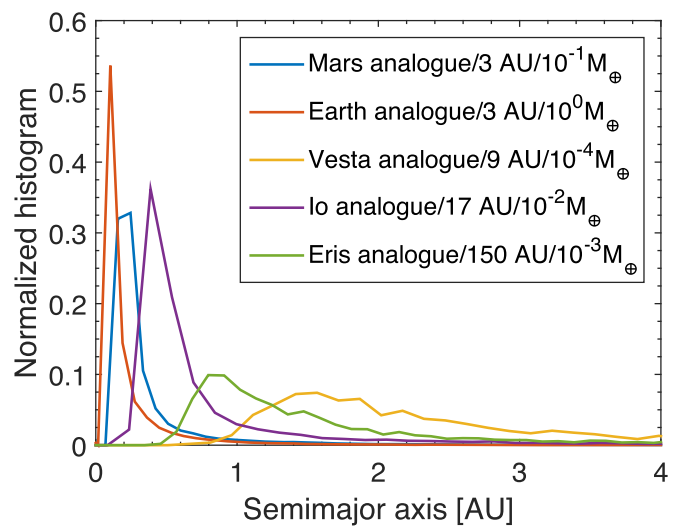

Figure 2. Semimajor axis normalized histogram of the final bound debris discs, formed by tidal disruption of several Solar system analogue planets/dwarf-planets, around a $0.6 \mathrm{M}_{\odot}$ WD. All have a similarly large pericentre of $q=1 \mathrm{R}_{\odot}$. Resolution is $200 \mathrm{~K}$ particles.

analytical model, we might expect complete symmetry, however, the histogram is normalized by the number of disrupted fragments. Unbound fragments (or 'escaped' in panel 1a) never return to disrupt further, whereas bound fragments do return, and are repeatedly dissected to smaller and smaller pieces (according to the analysis in Section 3.2) and thus increase in numbers. In panel 1(a), the disruption is very deep and the planet breaks into its constituent particles already during the first flyby. The relatively small number of fragments that return for subsequent disruptions cause the distribution to depart from symmetry, but the effect is small. In contrast, the bound debris discs in panels 1(b) and 1(c) are heavily processed by repeated disruptions, thus increasing fragment numbers. It is important to keep in mind that here the asymmetry is not indicative of the mass fraction of bound and unbound materials, which are approximately equal.

In Fig. 2, we further show all the debris discs from Table 1 that have a pericentre distance of $q=1 \mathrm{R}_{\odot}$, while the semimajor axes and masses vary. In spite of varying in semimajor axis, all of these simulations approximately satisfy the condition $r_{\text {crit }} \ll$ $R$, hence the cut-off should be proportional to $d^{2} / 2 R$, but smaller by some factor due to the large $1 \mathrm{R}_{\odot}$ pericentre distance (as we have discussed in detail for Fig. 1c). It is easily recognized that the innermost semimajor axis cut-off and the peak in each curve correlates (left to right) with the planet size/mass. The bigger the planet, the smaller $d^{2} / 2 R$. As previously mentioned, the innermost semimajor axis cut-off points correspond to the surface constituent particles (for which $r=R$ and $d^{2} / 2 R$ ); however, the peaks in the semimajor axis dispersion correspond to constituent particles within the planets (i.e. $r<R$ ). Mathematically, the distributions from peak to cut-off should therefore be narrower for the larger planets, as is clearly the case.

\subsection{Size distribution}

In principle the tidal disruption problem is determined from the density ratio of the planet and the star. The higher the (relative) planet density, the lower the Roche limit. If the object passes within the Roche limit the material disrupts. Setting aside other complicating aspects such as self-rotation, intrinsic material strength, and complex internal structures, if an object continues to pass within the Roche limit it should in principle continue to disrupt to smaller and smaller pieces until the latter finally reach a size in which the material strength dominates, preventing any further disruptions.

In our numerical simulations, however, the minimum size that can be attained is determined by the resolution. Even our largest resolution, which exceeds that of any previous tidal disruption study by approximately two orders of magnitude, still involves large single $\mathrm{SPH}$ particles of a characteristic size of at least a few $\mathrm{km}$. The limiting physical size of strength-dominated particles which can withstand being ripped apart by the tidal forces is however of the orders of tens of meters to hundreds of meters (Brown, Veras \& Gänsicke 2017), and perhaps even smaller. Therefore, our initial expectation is that eventually, our hybrid simulations would results in a flat size distribution - exactly the size of a single SPH particle.

In Fig. 3, we show how the fragment size distribution of a Mars analogue at 3 au indeed evolves towards the numerical minimum, as a function of time. Fragment radii are plotted as a function of their semimajor axis. Each fragment is depicted by a circle whose size is scaled with the number of its constituent SPH particles, and the colour coding represents different compositions, as in the underlying colourbar. In panel 3(a), we show the original Mars-like planet, with approximately the correct dimensions, and an orbit at precisely 3 au. Then, in panel 3(b) the planet undergoes its initial tidal breakup, disrupting and then fragmenting into multiple clumps with tight orbits, in agreement to Fig. 2, and one major bound fragment with a semimajor axis greater than that of the original planet. As we advance to the next panels there is a sequential flattening in the size distribution, proceeding from left to right. The last remaining major fragment is beyond $3 \mathrm{au}$, and thus it takes longer than 1 orbital period of the original planet before it can disrupt. After an additional orbit time, in panel 3(d), the evolution is nearly complete. Full completion is however reached only when the last bound, extremely eccentric fragment, returns to the tidal sphere one last time.

Note that at the end of the simulation in panel 3(f), not all the particles follow a single horizontal line, as might be expected. Instead there is one prominent line in addition to multiple others, that span a range of sizes, despite reaching the minimum single SPH particle limit. The reason for this behaviour is that the SPH particles span across a range of densities. When we inspect the data in detail, we observe that during this particular disc formation scenario many of the particles inhabit the cold expanded region in the Tillotson equation of state due to the pulling apart motion of the tidal stream, for the initial (major) tidal disruption. The cold expanded state in the Tillotson equation of state phase space is the region in which the density is lower than the reference density (at zero pressure) and the internal energy is lower than the energy of incipient evaporation. This region of the phase space is reached through tension rather than compression (see Burger, Maindl \& Schäfer 2018).

We also analyse other simulations and find that low particle densities may be reached through a different route - the vapourization or partial vapourization regimes - which occur when the internal energy surpasses the energy of complete or incipient vapourization, respectively. Such high internal energy states are not reached in the scenario presented in Fig. 3, however for an Earth-mass planet with the same orbital parameters, we do see some particles with internal energies above the energy of incipient vapourization. These particles gain their energy during the fragmentation phase, when the stream collapses under its own gravity and the compression in the outer layers of the largest fragments is sufficiently high. High internal energies are especially dominant in the more violent tidal disruptions with $q=0.1 \mathrm{R}_{\odot}$, however, in this case they occur at a much earlier stage when the planet passes through the tidal sphere. 


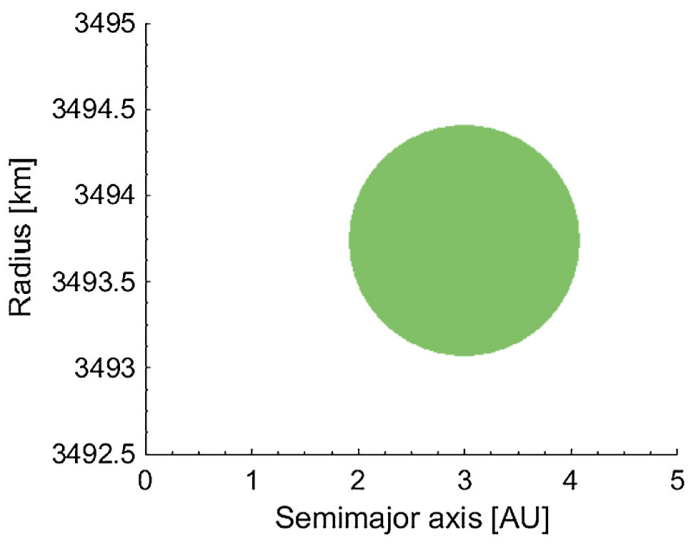

(a) $t=0$, before disruption.

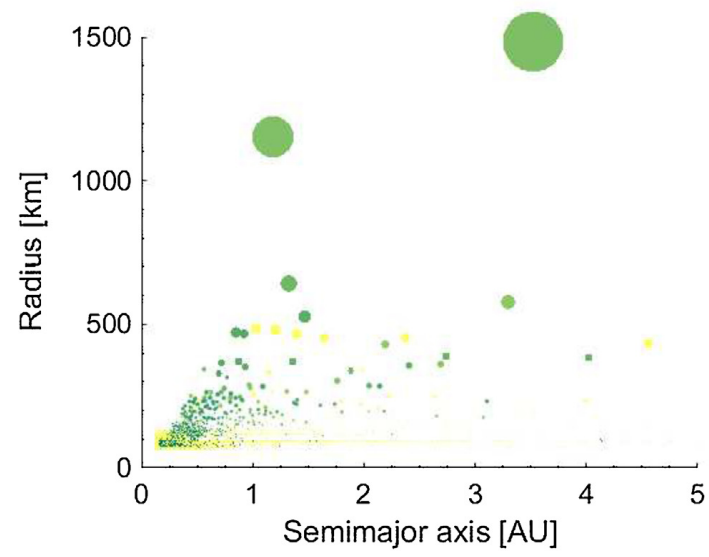

(c) $\mathrm{t}=0.2$ planet orbits.

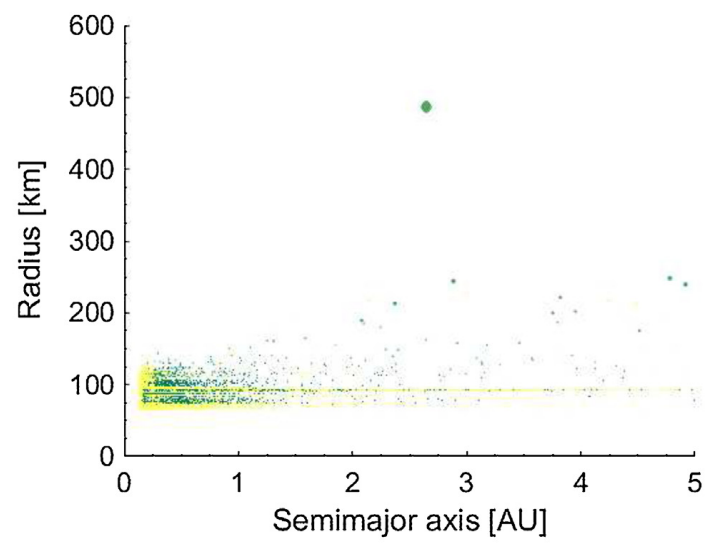

(e) $\mathrm{t}=2$ planet orbits.

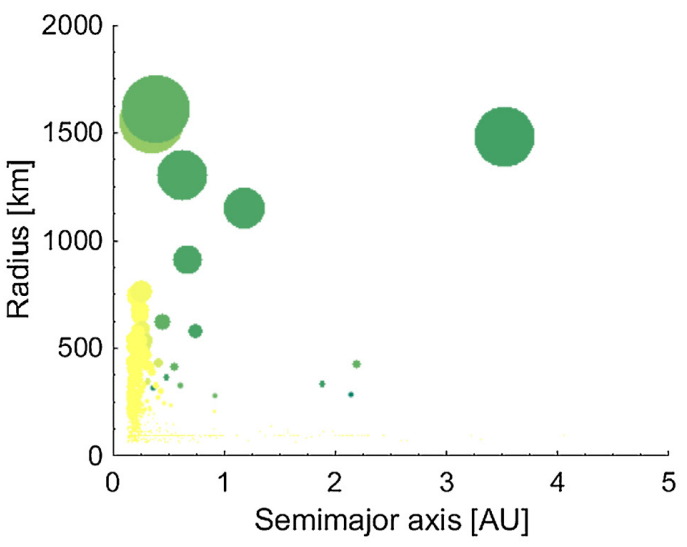

(b) $\mathrm{t}=0.02$ planet orbits.

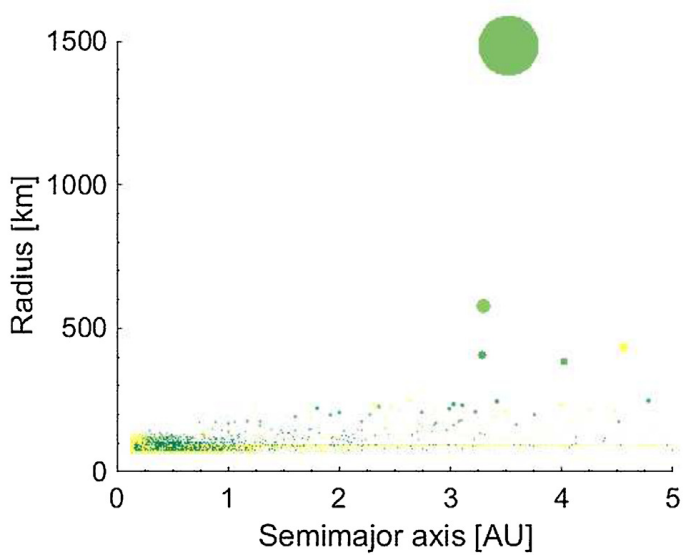

(d) $t=1$ planet orbits.

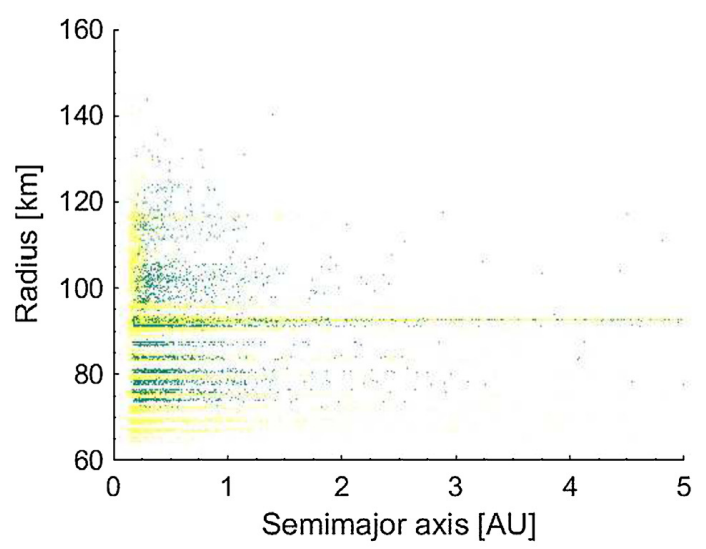

(f) $\mathrm{t}=11741$ planet orbits.

\section{Pure iron $\quad 50 \%$ mix $\quad$ Pure rock}

Figure 3. Temporal evolution of fragment size distribution of a tidally disrupted Mars analogue $\left(a=3\right.$ au and $\left.q=0.5 \mathrm{R}_{\odot}\right)$ around a $0.6 \mathrm{M}_{\odot}$ WD. Fragment radii are plotted as a function of their semimajor axis. Each fragment is depicted by a circle whose size is scaled with the number of its constituent SPH particles, and the colour coding represents different compositions (see underlying colourbar). Panels (a)-(f) depict the time progress in units of the original planet's orbital period, at $a=3$ au. After 11741 such orbital periods, the last remaining bound fragment disrupts.

\subsection{Formation time}

The typical outcome of tidal disruptions is to grind down a disrupted planet (bound disc) into smaller and smaller pieces. In our simulation suite, planets are large enough and orbit far from the star, and therefore fragments are often flagged to very tight orbits. It is also possible to find bound fragments that are flagged out to larger orbits than that of the original planet, however their fraction is typically very small, and depends on the exact parameters of the problem. By that logic, all the fragments interior to the original 
Table 2. Hybrid disc formation progress (per cent).

\begin{tabular}{lcccc}
\hline Hybrid model & $q\left(\mathrm{R}_{\odot}\right)$ & 1 Orbit & 2 Orbits & 5 Orbits \\
\hline Earth & 0.1 & 99.85 & 99.89 & 99.94 \\
& 0.5 & 96.91 & 98.79 & 99.21 \\
Mars & 1.0 & 53.41 & 73.99 & 91.71 \\
& 0.1 & 98.87 & 99.53 & 99.89 \\
& 0.5 & 88.12 & 96.89 & 99.78 \\
Main-belt(Vesta) & 1.0 & 10.46 & 55.43 & 98.54 \\
Satellite (Io) & 1.0 & 29.53 & 84.10 & 96.17 \\
Kuiper-belt(Eris) & 1.0 & 12.13 & 56.96 & 99.98 \\
& 1.0 & 36.70 & 74.31 & 100.0 \\
\hline
\end{tabular}

Note. The first two columns show the name and simulated pericentre distance of each analogue planet. The last three columns show the disc formation progress in per cent, i.e. the ratio between the ongoing number of bound fragments to the final number at the end of each simulation, as a function of the number of orbital periods (of the original planet) since the initial planet disruption.

semimajor axis are expected to re-disrupt before one orbit of the original planet passes. They are obviously much smaller than the original planet, and at least initially their size follows a power-law distribution (Section 3.2). Given their smaller size and typically much tighter orbits their subsequent disruptions are less dispersive than that of the original planet, but they nevertheless keep dissecting into smaller pieces, since their pericentre distance remains the same. Fig. 3 is a classic depiction of the aforementioned behaviour.

Now consider a simple exercise. A fragment disrupts into sub-fragments that follow a power-law size distribution. Further consider a limiting case wherein the largest sub-fragment is half the size of the original planet and has the same semimajor axis. In this scenario, the largest fragment would reach one thousandth of the size of the original fragment, within merely 10 orbital periods $\left(2^{10}\right)$. In fact, only the fragments that are initially flagged out to larger orbital periods than that of the original planet take a long time to disrupt and complete the formation of the disc. This is exactly what we see in various hybrid models (e.g. Fig. 3f).

By rule of thumb, therefore, the formation of the disc should be rapid and typically take only a few orbits of the original planet. This is indeed the outcome in all of our hybrid simulations. In Table 2, the disc formation progress for our hybrid models is shown as a function of time, or rather as a function of orbital periods of the original planet. Each row corresponds to a different hybrid simulation. The name is listed in the first column, the pericentre distance in the second, and then the progress in per cent denotes the ratio between the number of bound fragments at time $t$ to that at the end of the simulation.

In general, the rate of disc formation inversely correlates with the pericentre distance. Even in simulations in which the pericentre is the largest and close to the Roche limit, 5 orbits are sufficient in order to gain a considerable progress. In all simulations, the disc formation is at least 91 per cent complete by merely 5 orbital periods of the original planet. We note that in Table 2, the orbital period increases with each simulated planet (see Table 1), and therefore in absolute terms the progress in $t$ is slower.

\subsection{Self-rotation distribution}

We investigate the effect of tidal spin-up of fragments, with the caveat that as disc formation progresses, the size distribution flattens out, eventually dissecting all fragments to single SPH particles (see Section 3.2). For the latter, rotation cannot be calculated, and we can

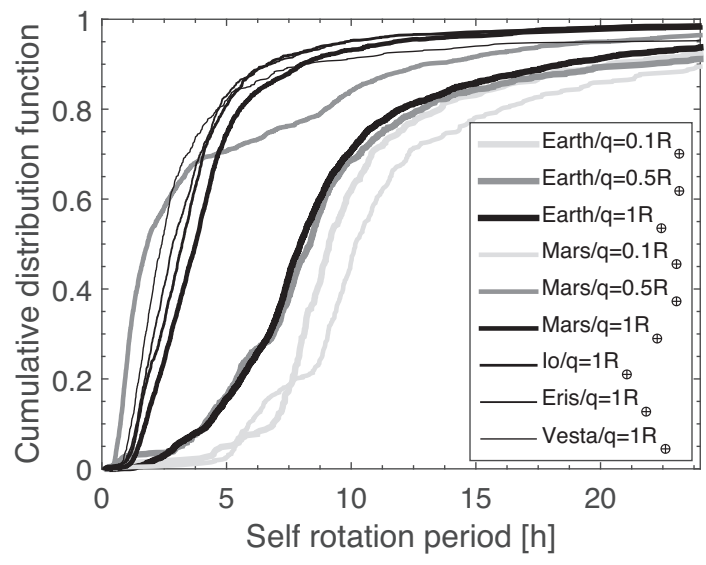

Figure 4. The cumulative distribution function of disc fragment rotation periods for all hybrid models in Table 1, after 1 orbital period of the original planet. The line thickness correlates with planet mass and its colour with pericentre distance, as shown in the legend. The majority of simulations show very rapid fragment rotation.

only postulate on the self-rotation properties of single SPH particles by extrapolation, relying on earlier epochs in which we still have large fragments in our disc. We nevertheless attempt to quantify the fragment rotational properties during the disc formation, because their rotation enhances tidal breakup (see related discussion in Paper I), could contribute to understanding phenomena like the Yarkovsky effect (see appendix of Paper I) and since fast-spinning fragments can be further spun up in a manner similar to that suggested by Makarov \& Veras (2019), if the Roche limit is effectively reduced owing to fragment internal strength.

In Fig. 4, we plot the cumulative distribution function of all our hybrid simulations to gain some statistical insights. We only calculate the orbital period for fragments with two or more SPH particles, using the method in Malamud et al. (2018), while single SPH particles do not provide any rotational information and are therefore ignored. Note that at the beginning of disc formation, we have small number statistics for the $q=1 \mathrm{R}_{\odot}$ simulations (the disruptions being partial), while for the $q=0.1 \mathrm{R}_{\odot}$ simulations, virtually all fragments are close to the single SPH particle size, even after the first planet disruption, hence we have a similar problem. Based on Table 2, we find 1 orbital period a suitable choice to minimize small number statistics, at least for the majority of simulations.

As indicated by the legend, the sub-plots differ such that the more massive planets are depicted by thicker lines. Additionally, the grey-scale tone correlates with the pericentre distance, such that the black colour corresponds to $q=1 \mathrm{R}_{\odot}$ simulations. The results indicate two major groupings. The Earth simulations and the $q=0.1 \mathrm{R}_{\odot}$ Mars simulations are in the first group, while the rest of the simulations are in the second group. Both groups are characterized by fast rotating fragments, however in the former group some $70-80$ per cent of the fragments have rotation periods less than merely twice the $2.2 \mathrm{~h}$ cohesionless asteroid spin-barrier (Pravec, Harris \& Michalowski 2002).

\subsection{Resolution dependence on convergence}

We test the effect of resolution on the simulation results. Our main goal is to investigate convergence in terms of the bound disc fragment semimajor axis distribution. 


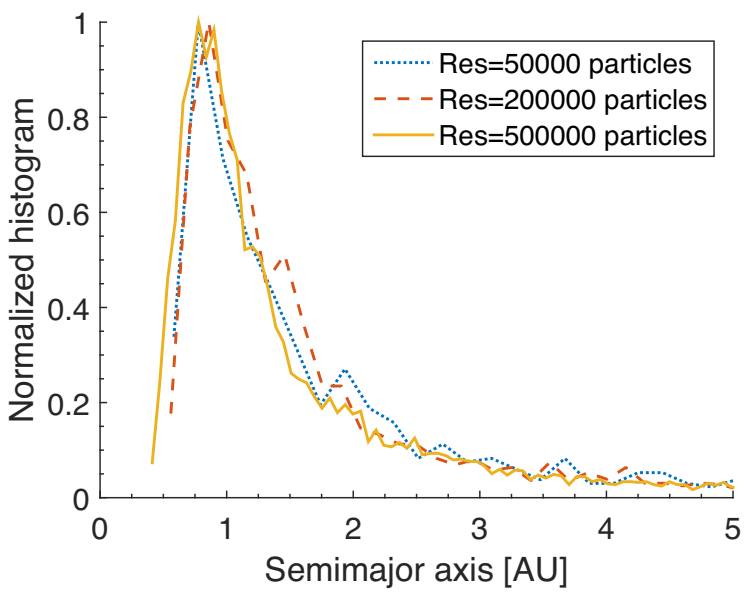

Figure 5. Resolution dependence, comparing the eventual debris disc of a tidally disrupted Kuiper-belt analogue ( $a=150$ au and $\left.q=1 \mathrm{R}_{\odot}\right)$ around a $0.6 \mathrm{M}_{\odot} \mathrm{WD}$. Three superimposed normalized histograms for the fragment semimajor axis are presented, given low $(50 \mathrm{~K} /$ dotted line), intermediate (200K/dashed line), and high $(500 \mathrm{~K} /$ solid line) number of SPH particles.

For our comparison we simulate the Kuiper-belt/Eris analogue case, where $a=150$ au and $q=1 \mathrm{R}_{\odot}$, in three different resolutions. In addition to our fiducial resolution value of $200 \mathrm{~K}$ SPH particles, we also produce two simulations with low $(50 \mathrm{~K})$ and high $(500 \mathrm{~K})$ resolutions. Fig. 5 then shows the normalized histograms of these three simulations (see legend), such that each histogram peaks at 1 , and their morphologies can be directly compared. We see that increasing the resolution has a relatively small impact. Thus, we conclude that the main characteristic of the disc, its semimajor axis distribution of fragments, is hardly affected by resolution.

We do not check resolution convergence for the disc fragment size distribution since the latter tends to be flat anyway, due to the continuous grinding down of all the bound debris to the level of smallest resolved single particles. Nevertheless, in Appendix, we show that fragments with a large pericentre distance (around $1 R_{\odot}$ ) and a small number of SPH particles $(<500)$ do not undergo the expected disruption and flattening of the size distribution, however, this behaviour is not physical but rather results from numerical issues. We show that it can be solved either by increasing the resolution or by decreasing the values of the artificial viscosity parameters. We thus conclude that the grinding down and flattening of the size distribution remain a valid physical interpretation.

\section{UNBOUND MATERIAL: EJECTION OF INTERSTELLAR ASTEROIDS}

\subsection{Size distribution}

As already mentioned, the scenarios in Table 1 are all characterized by typical $r_{\text {crit }}$ values much smaller than the object radius, which means that nearly half of the initial planet mass becomes unbound. The latter particles/fragments are ejected to the interstellar space, hence, unlike bound debris, they are not repeatedly dissected by subsequent disruptions to flatten out their size distribution. Instead, fragment sizes depend on the pericentre/breakup distance (see also Section 4.2). In all but the deepest disruptions, the emerging streams collapse under their own self-gravity to form large fragments from the debris. We thus expect a non-flat, and perhaps a power-law size distribution. In contrast, very deep disruptions violently break the

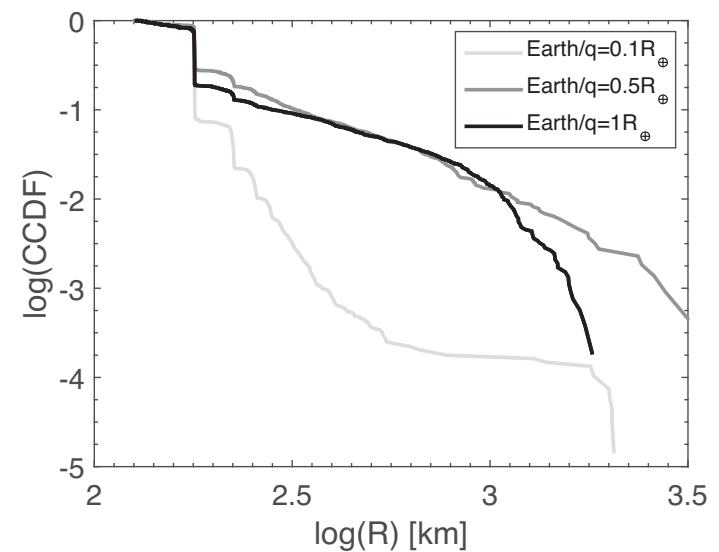

Figure 6. Comparing the size distribution of unbound material from a tidally disrupted Earth analogue $(a=3 \mathrm{au})$ around a $0.6 \mathrm{M}_{\odot} \mathrm{WD}$, with varying pericentre distances $\left(q=0.1-1 \mathrm{R}_{\odot}\right.$, correlating with grey-scale tone). The complementary cumulative distribution function is plotted as a function of size. Both axes are in logarithmic scale, and only the simulation with $q=0.5 \mathrm{R}_{\odot}$ approximately curves out a power-law distribution (linear correlation in $\log$ ).

planets into small, typically single SPH particles, and the emerging streams of debris do not collapse gravitationally to form larger fragments (see Section 1), hence we expect an outcome more closely resembling a flat distribution, with only a small number of fragments that coalesce from the denser part of the stream.

Indeed, Fig. 6 shows the simulation outcomes to broadly agree with these predictions. Here, we compare the unbound fragments that are ejected from a tidally disrupted Earth analogue around a $0.6 \mathrm{M}_{\odot} \mathrm{WD}$, for varying pericentre distances. The analysis is performed at the end of each simulation. Due to the similarity between the Earth and Mars cases (see Section 4.2), only the former is plotted here.

The complementary cumulative distribution function (or CCDF, the probability that fragment size is larger than $x$ ) is indicative of a power-law distribution when the axes are linearly correlated (both axes are plotted in logarithmic scale). Fig. 6 shows that only the simulation with $q=0.5 \mathrm{R}_{\odot}$ generates an approximately power-law distribution for the unbound fragments. A larger pericentre distance results in an almost identical distribution for fragments up to $10^{3} \mathrm{~km}$, however, larger fragments are more depleted compared to the former (See also panels $7 \mathrm{~b}$ and $7 \mathrm{c}$ ). This could be explained by the fact that for $q=0.5 \mathrm{R}_{\odot}$, the first flyby results in a full disruption, ejecting most of the unbound material. In contrast, for $q=1 \mathrm{R}_{\odot}$ the first few disruptions are partial, gradually ejecting smaller fragments from the rocky mantle first, and only later on the (much smaller) iron core disrupts entirely (see also Paper I for the discussion of a similar case, with full SPH simulation). Finally, for the $q=0.1 \mathrm{R}_{\odot}$ simulation we indeed get a much flatter size distribution, as expected.

In our simulation suite, only the Earth and Mars analogue cases have simulations with $q=0.5 \mathrm{R}_{\odot}$. From these cases we can extract the unbound fragments' size distribution power-law exponent (the CCDF slope subtracted by 1 ), which in both cases comes to approximately $4 / 3$.

\subsection{Self-rotation and composition distribution}

Unbound fragments are ejected to interstellar space at high speeds, and maintain their rotational properties, as they never again interact with their star of origin. In small, Oumuamua-like fragments 
(Meech et al. 2017), with irregular shapes, the angular momentum following a tidal disruption can be misaligned with the axis of maximum moment of inertia, so that the rotational velocity vector begins to precess and nutate (Scheeres et al. 2000). Such a tumbling motion has indeed been identified for Oumuamua, and given a monolithic, high-rigidity structure, its extremely long damping time-scale to return to principle-axis rotation could have kept it that way (Fraser et al. 2018). In contrast, resolution limitations in our study and the initial large size of the objects in Table 1, generate much larger fragments, ranging from large asteroids to dwarf-planet size. Our goal is therefore to study the rotation properties of the so called rogue dwarf-planets/asteroids that are flung out to interstellar space.

Fig. 7 is identical to Fig. 3, except that its $x$-axis denotes the fragment's orbital period, ranging from the cohesionless asteroid spinbarrier of 2.2-24 h (Pravec et al. 2002). As before, each unbound fragment is depicted by a circle whose size is scaled with the number of its constituent SPH particles, and the colour coding represents different compositions, as in the underlying colourbar. Therefore, this figure contains rotational, compositional, and size information for all unbound fragments, obtained after the tidal disruption of Earth and Mars analogue planets around a $0.6 \mathrm{M}_{\odot} \mathrm{WD}$, at the end of the simulation. Only these two planets have three different pericentre distances, which enables a more complete comparison.

From Fig. 7, we draw several conclusions. (a) Unbound fragment properties, including their rotation periods, composition, and relative (to-progenitor) sizes are very similar between the Earth (top row) and Mars (bottom row) cases, so at least for the unbound fragments this problem appears relatively scale-invariant. (b) Between various pericentre distances (columns), however, variations are easily noticeable. Physical differences in fragment formation and in the timing of their formation (see Section 4.1) completely change their properties. (c) In all panels, rotation and maximum fragment size inversely correlate. (d) Iron-composed (or mixtures with higherthan-rock bulk density) fragments generally rotate faster than purely rocky ones. The latter could be understood from breakup-velocity arguments. The breakup velocity is calculated from equating selfrotation and self-gravity, and we may express the fragment rotation period as a function of its density, such that $P_{\text {rot }}=\sqrt{3 \pi / G \rho}$, hence the inverse correlation.

\subsection{Velocity distribution}

The velocity distribution of ejected planetesimals is also of particular interest in regards to the recently observed interstellar objects going through the Solar system (Meech et al. 2017; de León et al. 2019) or meteors of interstellar origin (Siraj \& Loeb 2019). Moreover, such ejections could give rise to high velocity interstellar asteroids (or comets, although we do not consider icy compositions in this paper specifically), and their discovery can potentially pinpoint to such tidal disruption origin. We nevertheless note that unbound fragments produced in this paper, are larger than the two interstellar objects discovered in our Solar system thus far. Their small size can only be understood in the context of very deep tidal disruptions, or as second-generation fragments from intracollisions in the unbound tidal stream (see related discussion in Paper I).

In Figs 8-10, we show the detailed velocity distribution found for the ejected debris. As can be seen, ejection velocities could be highest for debris ejected from the disruption of the largest objects.

In particular, similar to the case of the ejection of hypervelocity stars following the tidal disruption of a binary star by a massive black hole (Yu \& Tremaine 2003), the ejection velocity should be of the order of:

$v_{\text {eject }}=\sqrt{2 v \delta v}$,

where $v$ is the velocity of the disrupted planet at the tidal breakup radius, and $\delta v$ is the extra velocity kick imparted to an ejected unbound fragment. Such $\delta v$ could be at most the escape velocity for the disrupted planet. For example, envision a fragment which is just marginally bound to the planet, or think about a maximally rotating planet - the velocity of the marginally bound fragment around the centre of mass of the planet is of the order of the escape velocity from that planet. Upon disruption the fragment velocity adds as a kick to the total velocity during a disruption, hence giving rise to equation (1) following the same argument in Yu \& Tremaine (2003).

Given the escape velocities of Earth, Mars, Io, Eris, and Vesta $\left(11.20,5.00,2.53,1.38,0.36 \mathrm{~km} \mathrm{~s}^{-1}\right.$, respectively), their densities $\left(5.50,3.92,3.53,2.52,3.42 \mathrm{~g} \mathrm{~cm}^{-3}\right.$, respectively) and taking the upper range of the likely tidal radius $r_{\mathrm{t}}(C=1.89$, e.g. Bear \& Soker 2013):

$\frac{r_{\mathrm{t}}}{\mathrm{R}_{\odot}}=C\left(\frac{M_{\mathrm{WD}}}{0.6 \mathrm{M}_{\odot}}\right)^{1 / 3}\left(\frac{\rho}{3 \mathrm{~g} \mathrm{~cm}^{-3}}\right)^{-1 / 3}$

one would then expect maximal ejection velocities of 93, 61, 43, 31, and $16 \mathrm{~km} \mathrm{~s}^{-1}$ respectively, nicely consistent with the maximal ejection velocities we find from the detailed simulations for encounters at $q=1 \mathrm{R}_{\odot}$. Note that even higher velocities can be attained at significantly deeper encounters; as discussed by Sari, Kobayashi \& Rossi (2010), these can provide for ejection velocities even up to $\sim 30$ per cent higher than those obtained from equation (1), consistent with our $q=0.1 \mathrm{R}_{\odot}$ simulations.

Planetesimals ejected at the lowest velocities of less than a few $\mathrm{km} \mathrm{s}^{-1}$, could be recaptured again into other planetary systems (see e.g. Grishin, Perets \& Avni 2019, Grishin \& Veras 2019) while ejection velocities $>\sim 60 \mathrm{~km} \mathrm{~s}^{-1}$ are significantly higher than would be expected for debris ejected from stars in the Galactic disc, and could therefore potentially point to a disruption origin, although high velocities could also be related to halo stars. An observed interstellar object with a high velocity whose direction could be traced to reside in the Galactic disc would provide a potential smoking gun signature.

\section{POSSIBLE IMPLICATIONS FOR THE ORIGIN OF TABBY'S STAR, ZTF J0139+5245 AND SDSS J1228+1040}

\subsection{Tabby's star - an ongoing disruption by an icy dwarf planet?}

KIC 8462852 (a.k.a Tabby's star) is an F type star which features extraordinary dips in its light curve, of up to 20 percent, first identified through the Kepler mission (Boyajian et al. 2016). Transit events last from a fraction of a day to several days, and the observed morphology of the dips vary considerably from case to case. Later observations suggested a periodicity of $4.31 \mathrm{yr}$ can be compatible with some of the observed transits (Sacco, Ngo \& Modolo 2018). The star lacks significant excess infrared emission (Thompson et al. 2016). To further the enigma of KIC8462852, Schaefer (2016) found evidence for secular dimming, with a 16 percent decrease in brightness from 1890 to 1980 . The Kepler data also showed similar evidence for long-term dimming, with 3 per cent over a $4 \mathrm{yr}$ time-scale (Montet \& Simon 2016).

Out of a multitude of hypotheses originally proposed (Boyajian et al. 2016), a favourite among several authors (Bodman \& Quillen 


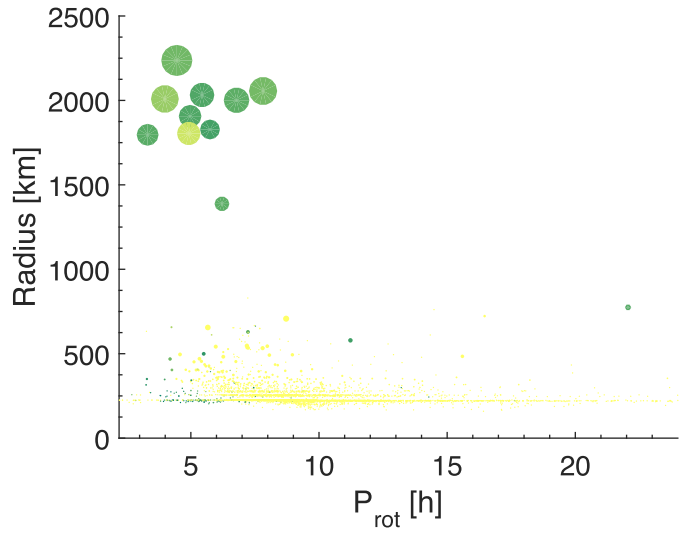

(a) Earth analougue, $q=0.1 R_{\odot}$.

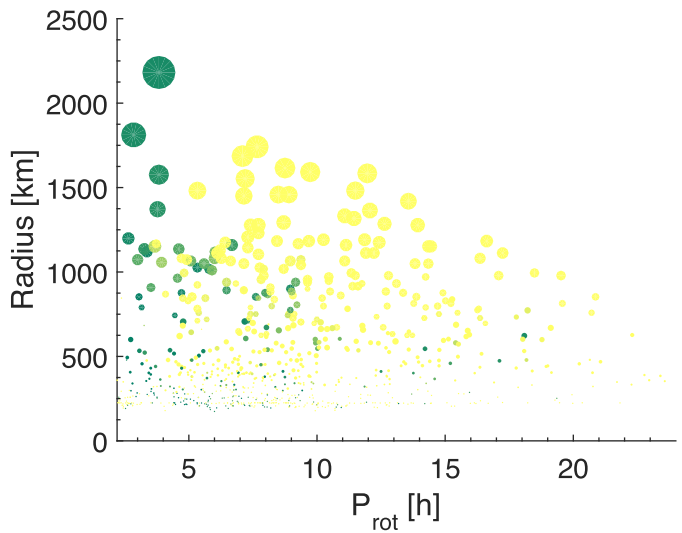

(c) Earth analougue, $q=1 R_{\odot}$.

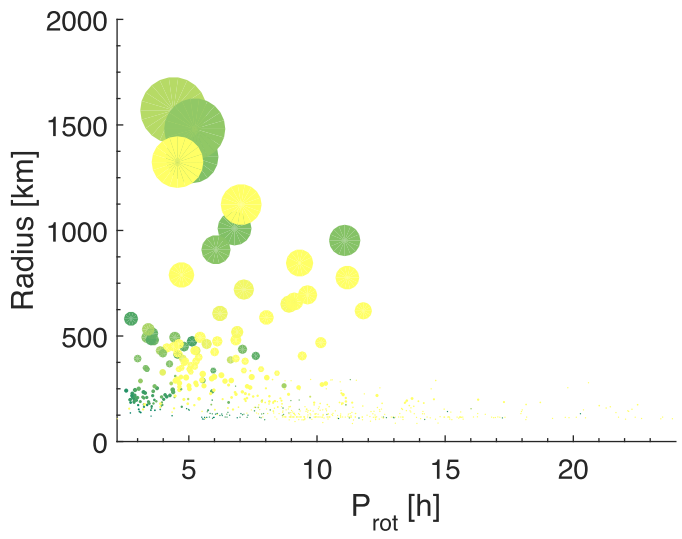

(e) Mars analougue, $q=0.5 R_{\odot}$.

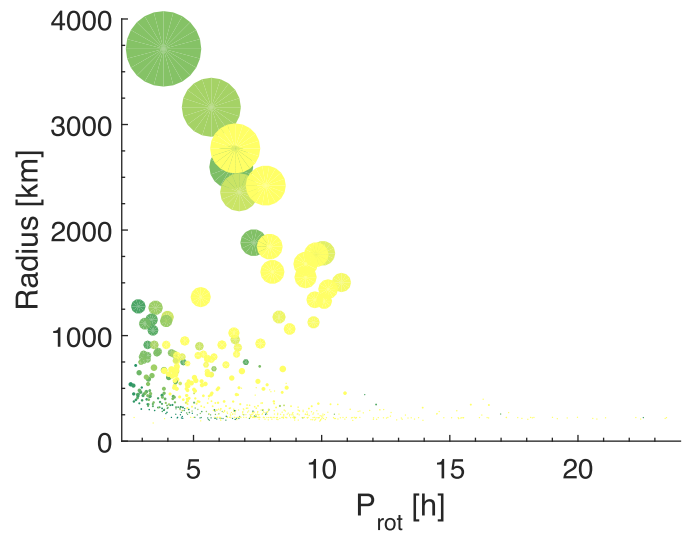

(b) Earth analougue, $q=0.5 R_{\odot}$.

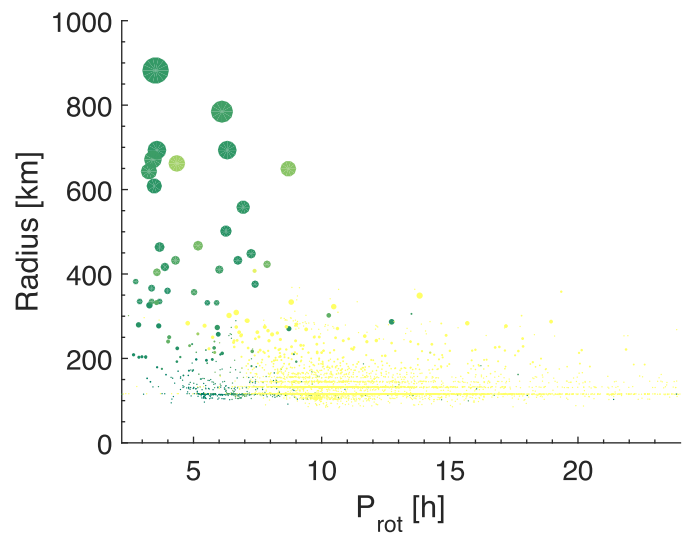

(d) Mars analougue, $q=0.1 R_{\odot}$.

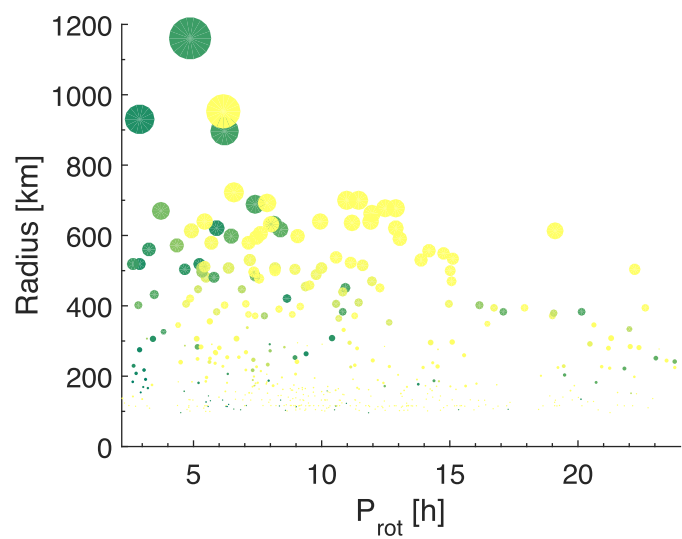

(f) Mars analougue, $q=1 R_{\odot}$.

\section{Pure iron $\quad 50 \%$ mix $\quad$ Pure rock}

Figure 7. Unbound fragment self-rotation distribution, of a tidally disrupted Earth (top row) and Mars (bottom row) analogues with $a=3$ au, and $q=0.1$ R $\odot$ (first column), $q=0.5 \mathrm{R}_{\odot}$ (second column) and $q=1 \mathrm{R}_{\odot}$ (third column), around a $0.6 \mathrm{M}_{\odot} \mathrm{WD}$. The fragment radii are plotted as a function of their rotation period. Each fragment is depicted by a circle whose size is scaled with the number of its constituent SPH particles, and the colour coding represents different compositions (see underlying colourbar). After 11471 such orbital periods, the last remaining bound fragment disrupts. 


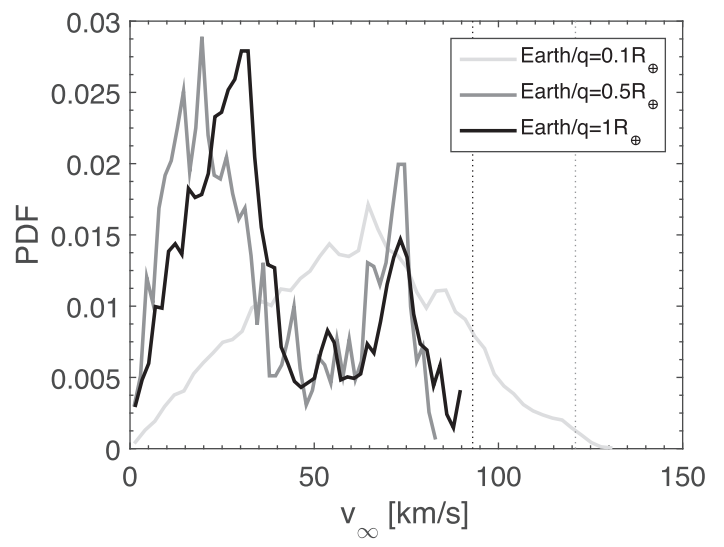

Figure 8. Comparing $V_{\infty}$ of unbound material from a tidally disrupted Earth analogue ( $a=3 \mathrm{au})$ around a $0.06 \mathrm{M}_{\odot} \mathrm{WD}$, with varying pericentre distances ( $q=0.1-1 \mathrm{R}_{\odot}$, correlating with grey-scale tone). The probability density function is plotted as a function of velocity. The maximal ejection velocities from analytical predictions are shown by dotted vertical lines (extremely deep disruptions increase the velocity by $\sim 30$ per cent and shown in grey colour).

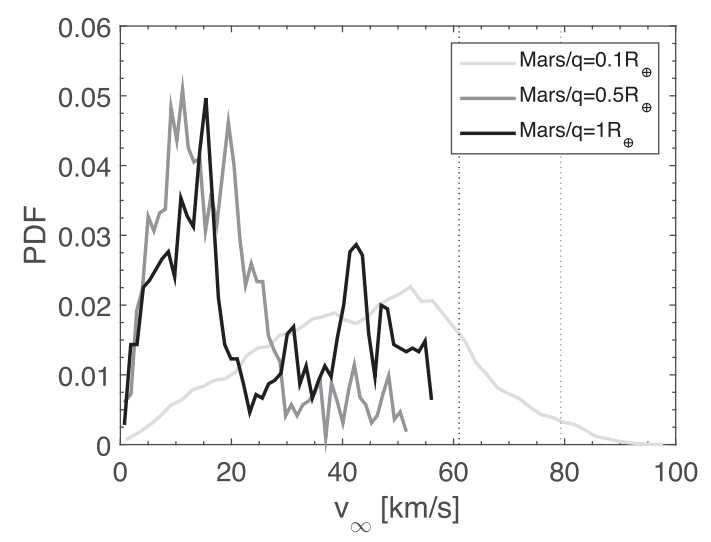

Figure 9. Comparing $V_{\infty}$ of unbound material from a tidally disrupted Mars analogue $(a=3 \mathrm{au})$ around a $0.6 \mathrm{M}_{\odot} \mathrm{WD}$, with varying pericentre distances $\left(q=0.1-1 \mathrm{R}_{\odot}\right.$, correlating with grey-scale tone). The probability density function is plotted as a function of velocity. The maximal ejection velocities from analytical predictions are shown by dotted vertical lines (extremely deep disruptions increase the velocity by $\sim 30$ percent and shown in grey colour).

2016; Neslušan \& Budaj 2017; Wyatt et al. 2018) was an exocomet origin. This scenario is deemed as potentially the most likely suggestion, although it is not trivial to conceive an origin for swarms (Bodman \& Quillen 2016) or larger clumps (Neslušan \& Budaj 2017) of comet-like material, while also explaining the secular dimming. Wyatt et al. (2018) suggest that the secular dimming might be explained by dusty material that is unevenly distributed around a single elliptical orbit, but they do not consider in detail the origin of this elliptical dust structure.

We suggest a potential solution perfectly compatible with our hybrid model (but considering a main-sequence star instead of a WD), and can self-consistently explain the origin of the comets as well as the secular dimming. Previous studies (Bodman \& Quillen 2016; Neslušan \& Budaj 2017) suggest that the mass of transiting material is around $10^{-2}-10^{-4} \mathrm{M}_{\oplus}$. A tidally disrupted parent object

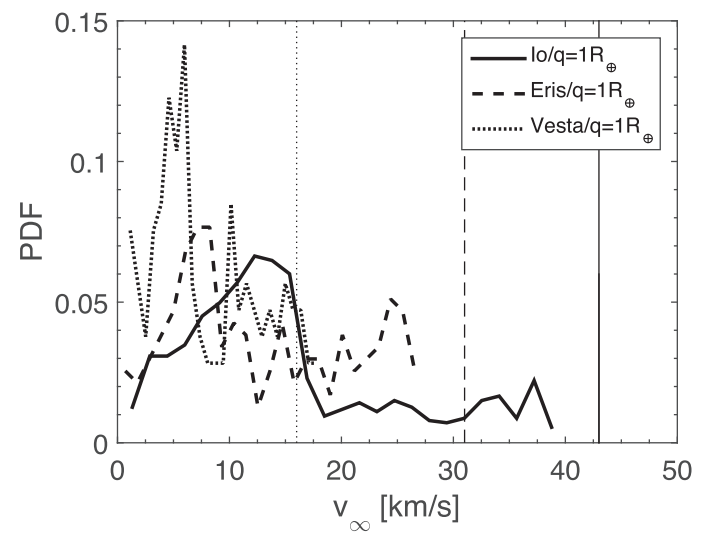

Figure 10. Comparing $V_{\infty}$ of unbound material from a tidally disrupted Io ( $a=17 \mathrm{au}$, solid line), Eris ( $a=150 \mathrm{au}$, dashed line), and Vesta ( $a=9 \mathrm{au,}$ dotted line) analogues around a $0.6 \mathrm{M}_{\odot} \mathrm{WD}$, with pericentre distance $q=$ $1 \mathrm{R}_{\odot}$. The probability density function is plotted as a function of velocity. The maximal ejection velocities from analytical predictions are shown by same-style vertical lines.

with an originally eccentric orbit and compatible mass, could tidally disrupt and form smaller sub-fragments of active nuclei, spewing gas and dust. An original orbit of several au is consistent with a non-dispersive disruption, given $r_{\text {crit }}$ of the order of $\sim 10^{3} \mathrm{~km}$, so it might generate a ring-like debris disc. An original orbit of several tens of au is consistent with a bi-modal disruption, given $r_{\text {crit }}$ of order $\sim 10^{1} \mathrm{~km}$, so it might generate a dispersed disc of fragments, peaking at a few au (as in the Vesta curve in Fig. 2). Both configurations are therefore potentially compatible with a swarm of eccentric fragments concentrated around 3 au (for which the corresponding orbit is tentatively around the $4.31 \mathrm{yr}$ periodicity reported by Sacco et al. 2018).

The only caveat is that the density of the original parent object cannot be too large, otherwise it would not tidally disrupt at distances larger than the star's circumference. Additionally, note that the typical separations between Roche radius and star circumference are much lower for main-sequence stars than they are for WDs. Another possibility is partial disruption of only the outer layers of a differentiated object [like in Veras et al. (2017)]. Thermo-physical models and observations from the Solar system predict such differentiated structures to be common, and the outermost layers could be composed of a mixture of ice and rock (e.g. see Malamud \& Prialnik 2015; Malamud, Perets \& Schubert 2017).

Finally, the secular dimming is a natural consequence of the hybrid model, as the number density of fragments increases as a function of time. While swarms of larger objects produce the large transit events, the overall flux of smaller debris in the disc increases on a time-scale that is proportional to the orbit of the major fragments/original planet, and produces a gradual dimming of the star in a quasi-steady-state like manner.

The detailed study of this possibility will be explored elsewhere, through the investigation of various plausible scenarios along the previously mentioned lines. We hypothesize that this explanation could be explored as a general solution for an entire class of similar dipper stars. Schaefer (2019) shows that the other dippers do not exhibit evidence for secular dimming, however, they also display much shallower transits. It could be that KIC 8462852 simply captures the phenomena at a much earlier stage. 


\subsection{ZTF J0139+5245 - an early stage asteroid disruption} around a white dwarf?

In a recent paper, Vanderbosch et al. (2019) identify the second WD for which repeated transits have been observed. In contrast to the more widely known WD $1145+017$, here the transits duration is much longer, of the order of several weeks, and the inferred periodicity is around $110 \mathrm{~d}$.

According to the analysis of Vanderbosch et al. (2019), the likely distance of closest approach could imply an eccentricity larger than 0.97 , and a maximum orbital separation of 0.72 au. At periastron, a transiting grain would take $\sim 1$ min to transit, and as much as 1-2 $\mathrm{h}$ if at apastron. The much longer transits observed, imply the presence of an extended stream of disrupted debris.

Based on our current study, we deem the possibility of a bi-modal type tidal disruption less likely than that of a non-dispersive tidal disruption of a small asteroid. The latter is expected to generate a coherent ring of debris with well-separated transits, as seems to be the case here, whereas the former might involve a multitude of fragments with very different timings (like in Fig. 2). The transits duration is also compatible with a non-dispersive ring of debris, since numerical simulations in both the Veras et al. (2014) study and in our validation tests in Paper I clearly show the gradual lengthening of the tidal stream prior to completely filling out the ring.

If this interpretation is correct, we are witnessing evidence for an ongoing tidal disruption at a rather early stage of its evolution. Our models predict that the ring should fill out at time-scales proportional to the orbit, and since the orbit is not exceedingly long in this case, we might even predict that the entire filling of the disc could be monitored within a few decades.

For modelling the ZTF J013906.17+524536.89 transits, timestep limitations make full SPH or $\mathrm{N}$-body simulations such as that of Veras et al. (2014) computationally difficult, if not impossible, certainly at high resolutions. With the hybrid model, however, we are not limited by the relatively large orbit, and these issues can be explored in depth in a future study.

\subsection{Producing the possible intact planet core of SDSS J1228+1040?}

The popular exoplanet-searching transit method has proven observationally difficult when used to search for planets around WDs, given how faint they typically are. Recently, Manser et al. (2019) have used short-cadence spectroscopy in order to search for signs of random variations in the gaseous emission of a WD (SDSS J122859.93+104032) with a known gaseous disc, which they hypothesized would be produced by random gas-generating collisions among particles of dust. Instead they found periodic variations, which led the team to conclude that the emissions result from an excited cloud of gas trailing a metallic object up to a few hundred $\mathrm{km}$ in size, with a two-hour orbital period. Or else, the planetesimal itself is producing the gas since its orbit is close enough to the star (especially if it is eccentric), triggering surface sublimation. This discovery may turn out to become an additional important method for detecting planetary material.

How might one produce a large intact iron object with the size of up to several hundred $\mathrm{km}$, and an orbit with a pericentre distance ranging from 0.34 (if eccentric) to $0.73 R_{\odot}$ (if circular), is an outstanding question (Manser et al. 2019), and it is suggested that this object could be a left over planet core. Clearly, it must have reached that part of the disc after the WD itself formed. The detailed formation simulation studies performed until now (Veras et al. 2014,
2017) have considered neither the correct sizes nor compositions. Our study has investigated for the first time the kind of parent objects that may indeed give rise to such planetary cores. Nevertheless, none of the planets that we have simulated has an intact surviving core. The mitigating circumstances could be that our simulated pericentre distances were insufficiently large to allow for the core survival by means of its higher density alone. More likely, we would require to repeat our hybrid simulations albeit utilizing SPH formulations that incorporate internal strength. Previous studies tentatively argue that an object held together by internal strength could indeed avoid disruption (Bear \& Soker 2013; Brown et al. 2017; Manser et al. 2019). The hybrid model therefore has the potential to identify the most likely parent-objects leading to the initial formation of a comparable iron core and its accompanying disc, which would later evolve to give rise to the SDSS J122859.93+104032 observables.

\section{SUMMARY}

In this study, we employed a novel hybrid model introduced in an accompanying paper (Paper I) to study the typical debris discs that emerge from tidal disruptions of rocky dwarf and terrestrial planets, covering a range of masses, pericentre distances, and semimajor axes between 3 and 150 au. For the scenarios mentioned above, the resulting semimajor axis distribution at the end of disc formation bears a close resemblance to a bi-modal disruption distribution, produced after the initial flyby of the disrupted progenitor. However, the departure from the analytical impulse approximation distribution is apparent when the pericentre distance is either a small ( $<10$ per cent) fraction of the Roche limit or alternatively close to the Roche limit, which could be explained by major departures from the aforementioned impulse approximation assumptions. Our innermost orbit for the disc semimajor axis dispersion is approximately constant (to a factor of a 2), validating similar results for stellar tidal disruptions that also refuted the 'frozen-in' approximation in the energy spread of bound debris (Guillochon \& Ramirez-Ruiz 2013; Stone et al. 2013; Steinberg et al. 2019).

We also show that repeated disruptions in the bound debris disc eventually flatten out the power-law size distribution of each individual disruption, finally settling on the numerical minimum of a single SPH particle. The time-scale of disc formation completion in our hybrid models is compatible with that found in our full SPH simulations in Paper I. All of our hybrid simulations are 91100 percent complete in just five orbital periods of the original progenitor. Tidal-spin-up is also consistent with the full SPH simulations, although here we statistically quantify the phenomena. As much as $70-80$ per cent of the fragments have rotation periods less than twice the known $2.2 \mathrm{~h}$ cohesionless asteroid spin-barrier. Resolution convergence in semimajor axis distribution is deemed relatively unimportant between $50 \mathrm{~K}$ and $500 \mathrm{~K}$ SPH particles.

Finally, the unbound mass fraction is around 50 percent for all the disruptions modelled here. We characterize the properties of such ejected debris that then become interstellar asteroids, possibly similar to the recently observed 'Oumuammua' and 'Borisov' interstellar objects coming through the Solar system. Unbound fragments follow a power-law size distribution with an $\sim 4 / 3$ exponent, and we show that iron core fragments rotate faster than rocky fragments. We also find that tidal disruption of massive Earthmass objects can eject very high velocity (up to several times the Sun's relative velocity) interstellar objects, which might provide a smoking gun signature for their origin.

Finally, we briefly discuss how our results, and in particular the bi-modal regime of tidal disruptions which might help explain the 
origins of Tabby's star variability, the transits of ZTF J0139+5245 and possible surviving planetary core around SDSS J122859+1040 from disruptions of dwarf planets.

\section{ACKNOWLEDGEMENTS}

We wish to thank the anonymous reviewer for excellent suggestions and comments that have greatly improved this manuscript. UM and HBP acknowledge support from the Minerva center for life under extreme planetary conditions, the Israeli Science and Technology ministry Ilan Ramon grant and the Israel Sience Foundation (ISF) I-CORE grant 1829/12.

\section{REFERENCES}

Bear E., Soker N., 2013, New Astron., 19, 56

Bergfors C., Farihi J., Dufour P., Rocchetto M., 2014, MNRAS, 444, 2147

Bodman E. H. L., Quillen A., 2016, ApJ, 819, L34

Bonsor A., Mustill A. J., Wyatt M. C., 2011, MNRAS, 414, 930

Boyajian T. S. et al., 2016, MNRAS, 457, 3988

Brown J. C., Veras D., Gänsicke B. T., 2017, MNRAS, 468, 1575

Burger C., Maindl T. I., Schäfer C. M., 2018, Celest. Mech. Dyn. Astron., 130,2

Caiazzo I., Heyl J. S., 2017, MNRAS, 469, 2750

de León J., Licandro J., Serra-Ricart M., Cabrera-Lavers A., Font Serra J.,

Scarpa R., de la Fuente Marcos C., de la Fuente Marcos R., 2019, Res.

Notes Am. Astron. Soc., 3, 131

Debes J. H., Sigurdsson S., 2002, ApJ, 572, 556

Debes J. H., Walsh K. J., Stark C., 2012, ApJ, 747, 148

Dennihy E., Clemens J. C., Dunlap B. H., Fanale S. M., Fuchs J. T., Hermes J. J., 2018, ApJ, 854, 40

Desharnais S., Wesemael F., Chayer P., Kruk J. W., Saffer R. A., 2008, ApJ, 672,540

Doyle A. E., Young E. D., Klein B., Zuckerman B., Schlichting H. E., 2019, Science, 366, 356

Dufour P. et al., 2007, ApJ, 663, 1291

Farihi J., 2016, New Astron. Rev., 71, 9

Fraser W. C., Pravec P., Fitzsimmons A., Lacerda P., Bannister M. T., Snodgrass C., Smolić I., 2018, Nat. Astron., 2, 383

Gänsicke B. T., Koester D., Farihi J., Girven J., Parsons S. G., Breedt E., 2012, MNRAS, 424, 333

Grishin E., Veras D., 2019, MNRAS, 489, 168

Grishin E., Perets H. B., Avni Y., 2019, MNRAS, 487, 3324

Guillochon J., Ramirez-Ruiz E., 2013, ApJ, 767, 25

Hamers A. S., Portegies Zwart S. F., 2016, MNRAS, 462, L84

Harrison J. H. D., Bonsor A., Madhusudhan N., 2018, MNRAS, 479, 3814

Hollands M. A., Gänsicke B. T., Koester D., 2018, MNRAS, 477, 93

Jura M., 2003, ApJ, 584, L91

Jura M., 2008, AJ, 135, 1785

Jura M., Young E. D., 2014, Ann. Rev. Earth Planet. Sci., 42, 45

Jura M., Farihi J., Zuckerman B., Becklin E. E., 2007, AJ, 133, 1927

Jura M., Farihi J., Zuckerman B., 2009, AJ, 137, 3191

Kilic M., von Hippel T., Leggett S. K., Winget D. E., 2006, ApJ, 646, 474

Klein B., Jura M., Koester D., Zuckerman B., Melis C., 2010, ApJ, 709, 950

Koester D., Gänsicke B. T., Farihi J., 2014, A\&A, 566, A34

Kratter K. M., Perets H. B., 2012, ApJ, 753, 91

Makarov V. V., Veras D., 2019, ApJ, 886, 127

Malamud U., Perets H. B., 2020, MNRAS, 492, 5561

Malamud U., Prialnik D., 2015, Icarus, 246, 21

Malamud U., Perets H. B., Schubert G., 2017, MNRAS, 468, 1056

Malamud U., Perets H. B., Schäfer C., Burger C., 2018, MNRAS, 479, 1711

Manser C. J. et al., 2016, MNRAS, 455, 4467

Manser C. J. et al., 2019, Science, 364, 66

Meech K. J. et al., 2017, Nature, 552, 378

Metzger B. D., Rafikov R. R., Bochkarev K. V., 2012, MNRAS, 423, 505

Michaely E., Perets H. B., 2014, ApJ, 794, 122
Montet B. T., Simon J. D., 2016, ApJ, 830, L39

Mustill A. J., Villaver E., 2012, ApJ, 761, 121

Neslušan L., Budaj J., 2017, A\&A, 600, A86

Payne M. J., Veras D., Holman M. J., Gänsicke B. T., 2016, MNRAS, 457, 217

Payne M. J., Veras D., Gänsicke B. T., Holman M. J., 2017, MNRAS, 464, 2557

Perets H. B., Gualandris A., 2010, ApJ, 719, 220

Perets H. B., Kratter K. M., 2012, ApJ, 760, 99

Petrovich C., Muñoz D. J., 2017, ApJ, 834, 116

Pravec P., Harris A. W., Michalowski T., 2002, Asteroid Rotations, University of Arizona Press, Tucson. p. 113

Rafikov R. R., 2011, MNRAS, 416, L55

Reach W. T., Kuchner M. J., von Hippel T., Burrows A., Mullally F., Kilic M., Winget D. E., 2005, ApJ, 635, L161

Reach W. T., Lisse C., von Hippel T., Mullally F., 2009, ApJ, 693, 697

Rickman H., Fouchard M., Froeschlé C., Valsecchi G. B., 2008, Celest. Mech. Dyn. Astron., 102, 111

Sacco G., Ngo L. D., Modolo J., 2018, J. Am. Assoc. Var. Star Obs. (JAAVSO), 46, 14

Sari R., Kobayashi S., Rossi E. M., 2010, ApJ, 708, 605

Schaefer B. E., 2016, ApJ, 822, L34

Schaefer B. E., 2019, Res. Notes Am. Astron. Soc., 3, 77

Schäfer C., Riecker S., Maindl T. I., Speith R., Scherrer S., Kley W., 2016, A\&A, 590, A19

Scheeres D. J., Ostro S. J., Werner R. A., Asphaug E., Hudson R. S., 2000, Icarus, 147,106

Shappee B. J., Thompson T. A., 2013, ApJ, 766, 64

Siraj A., Loeb A., 2019, preprint (arXiv:1904.07224)

Smallwood J. L., Martin R. G., Livio M., Lubow S. H., 2018, MNRAS, 480, 57

Steinberg E., Coughlin E. R., Stone N. C., Metzger B. D., 2019, MNRAS, 485, L146

Stephan A. P., Naoz S., Zuckerman B., 2017, ApJ, 844, L16

Stone N., Sari R., Loeb A., 2013, MNRAS, 435, 1809

Stone N., Metzger B. D., Loeb A., 2015, MNRAS, 448, 188

Swan A., Farihi J., Koester D., Holland s M., Parsons S., Cauley P. W., Redfield S., Gänsicke B. T., 2019, MNRAS, 490, 202

Thompson M. A. et al., 2016, MNRAS, 458, L39

Vanderbosch Z. et al., 2019, preprint (arXiv:1908.09839)

Vanderburg A. et al., 2015, Nature, 526, 546

Veras D., 2016, R. Soc. Open Sci., 3, 150571

Veras D., Gänsicke B. T., 2015, MNRAS, 447, 1049

Veras D., Leinhardt Z. M., Bonsor A., Gänsicke B. T., 2014, MNRAS, 445, 2244

Veras D., Leinhardt Z. M., Eggl S., Gänsicke B. T., 2015, MNRAS, 451, 3453

Veras D., Carter P. J., Leinhardt Z. M., Gänsicke B. T., 2017, MNRAS, 465, 1008

Villaver E., Livio M., Mustill A. J., Siess L., 2014, ApJ, 794, 3

Wolff B., Koester D., Liebert J., 2002, A\&A, 385, 995

Wyatt M. C., van Lieshout R., Kennedy G. M., Boyajian T. S., 2018, MNRAS, 473, 5286

Yu Q., Tremaine S., 2003, ApJ, 599, 1129

Zuckerman B., Koester D., Reid I. N., Hünsch M., 2003, ApJ, 596, 477

Zuckerman B., Melis C., Klein B., Koester D., Jura M., 2010, ApJ, 722, 725

\section{APPENDIX: RESOLUTION EFFECTS ON DISC SIZE DISTRIBUTION}

In the main text, we discuss the gradual flatenning of the bound disc fragment size distribution. All of our simulations for $q=0.1$ and $q=0.5 \mathrm{R}_{\odot}$ have indeed shown a similar pattern of behaviour, which over time reduces the size of tidally disrupted particles down to the numerical minimum. This sequence proceeds gradually from the inside out. Each disruption generates in itself a size distribution which roughly follows a power-law behaviour, but after many 
subsequent disruptions the distribution becomes flat. For $q=1 \mathrm{R}_{\odot}$, the outcome of the hybrid model is however not identical to those with $q=0.5$ and $q=0.1 \mathrm{R}_{\odot}$. Even partial disruptions should, eventually, strip material bit by bit until reaching the minimum size. In Fig. A1, however, we show an example with a different outcome. Here, we simulate a Kuiper-belt analogue at $a=150$ au and $q=1 \mathrm{R}_{\odot}$, using the hybrid model. All other models with $q=1 \mathrm{R}_{\odot}$ qualitatively result in the same outcome as Fig. A1. The plot shows the size distribution of the fragments at the end of the simulation. Fragments that are as large as 150-200 km and have relatively tighter orbits compared with the original object, are passing within the tidal sphere without being disrupted, which eventually leads to the cessation of the simulation.

This result is puzzling, since we expect the disruptions to continue, and the distribution to flatten, as previously mentioned. Here, we report that the reason for this outcome is numerical, and indeed not physical, as follows. Given the initial resolution of the hybrid simulation of 200K SPH particles, the number of SPH particles comprising even the largest of the fragments in Fig. A1 does not exceed 500. This low-resolution seems to have a significant effect when modelling mild disruptions with a large pericentre distance. In order to test the hypothesis that a higher resolution would produce a more accurate outcome in this regime, we take a characteristic fragment from the inner annulus in Fig. A1, i.e. with characteristic values $R=150 \mathrm{~km}, a=0.75 \mathrm{au}, q=1 \mathrm{R}_{\odot}$, and a pure rocky composition. We model this fragment's tidal evolution with full SPH simulations (as done in Paper I), but for only one flyby, using three different resolutions: $0.5 \mathrm{~K}, 5 \mathrm{~K}$, and $50 \mathrm{~K}$ SPH particles. In other words, we test a fragment resolution similar to that of the hybrid model, as well as one-order and two-orders of magnitude greater. The outcomes are shown in Fig. A2.

The relative sizes of the constituent SPH particles are easily noticeable in the first panel 12(a), in which the time corresponds to a position outside the Roche limit. Each resolution is also depicted by a different colour: dark blue for the lowest resolution, blue for the intermediate resolution, and light-blue for the highest resolution. Already at this early stage, we see a significant difference between the outcomes of each simulation, whereby the fragment distension

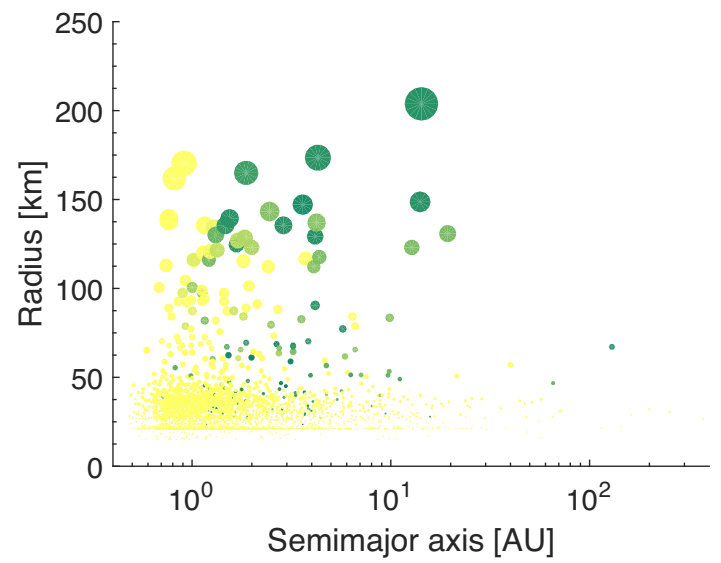

Figure A1. Fragment size distribution after the complete disc formation using the hybrid model, for a tidally disrupted Kuiper-belt dwarf-planet analogue $\left(a=150\right.$ au and $\left.q=1 \mathrm{R}_{\odot}\right)$ around a $0.6 \mathrm{M}_{\odot}$ WD. Fragment radii are plotted as a function of their semimajor axis. Each fragment is depicted by a circle whose size is scaled with the number of its constituent $\mathrm{SPH}$ particles, and the colour coding represents different compositions (see underlying colourbar).

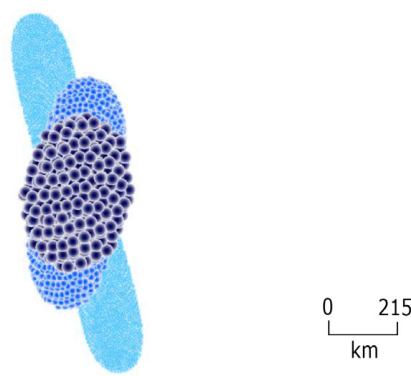

(a) $\mathrm{t}=13000 \mathrm{~s}$.
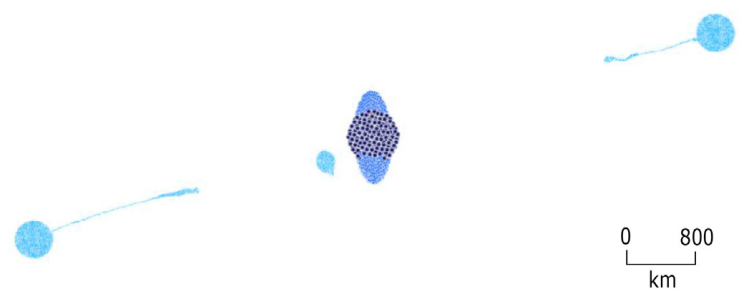

(b) $\mathrm{t}=62000 \mathrm{~s}$.

Figure A2. A top-down snapshot of partial tidal disruptions of three super imposed pure rocky fragments with $a=0.75$ au and a pericentre distance of $q=1 \mathrm{R}_{\odot}$, around a $0.6 \mathrm{M}_{\odot} \mathrm{WD}$. The fragments differ only in resolution: having $0.5 \mathrm{~K}$ (dark-blue), $5 \mathrm{~K}$ (blue) and $50 \mathrm{~K}$ (light-blue) $\mathrm{SPH}$ particles, respectively. The disruption outcome is shown (a) after the initial tidal encounter outside the Roche limit, and (b) after the fragmentation phase. Only the high resolution simulation results in the expected outcome of tidal breakup into three sub-fragments.

clearly correlates with the number of its constituent SPH particles. The second panel 12(b) captures the stream subsequent to the nearcompletion of the fragmentation phase, following its gravitational collapse. However, this fragmentation actually occurs only in the highest resolution simulation, splitting the original object into three separate sub-fragments (two narrow residual streams are in the process of conjoining with the right and left sub-fragments), while the two other lower resolution simulations show no sign of similar behaviour, and the original object remains whole.

We conclude that for tidal disruption simulations with a large pericentre distance, near the Roche limit, the resolution must be sufficiently high in order to generate physically plausible results, or else the SPH code requires some other modification. This issue never manifests itself in any of our simulations with pericentre distances of $q=0.5 \mathrm{R}_{\odot}$ or lower, regardless of resolution. Previous similar studies (Veras et al. 2014, 2017) have reported no dependence at all, for any value of $q$, for resolutions beyond merely $\sim 1000$ particles, however these authors utilize a very different $N$-body numerical model. This provides a hint as to the possible cause.

We have found that a possible solution to this problem is to lower the artificial viscosity parameters in the SPH model. We postulate that under certain conditions, the artificial viscosity, if allowed to operate unchecked, does not operate only when particle convergence occurs, but also acts to slow down neighbouring particles as they overtake because of differential rotation. We have successfully verified that lowering the artificial viscosity parameters by a factor of two fixes the problem in the intermediate resolution simulation. 
Lowering them by a factor of 4 , further fixes the problem even in the lowest resolution simulation. We however remain cautious with regard to the possibility of systematically reducing artificial viscosity in the SPH model (currently we implement fiducial $\alpha$ and $\beta$ values of 1 and 2 , respectively, as typically suggested by Schäfer et al. 2016), and propose that this merits future investigation of various artificial viscosity treatments.

How might these issues affect the hybrid model interpretation of results? Conceptually speaking, they are of little consequence. We propose that for the size distribution outcomes of simulations with $q \leq 0.5 \mathrm{R}_{\odot}$ there is no effect at all. When $q$ nears the Roche limit, we must simply postulate a flat distribution outcome, rather than rely on the hybrid model final outcomes, since our ability to increase the resolution indefinitely is unrealistic, and the artificial viscosity treatment requires additional studies. Our investigation in Fig. A2, however, proves that the flat distribution outcome is physically sound.

This paper has been typeset from a $\mathrm{T}_{\mathrm{E}} \mathrm{X} / \mathrm{L} \mathrm{T} \mathrm{T}_{\mathrm{E}} \mathrm{X}$ file prepared by the author. 